\title{
Laparoscopy-assisted versus enteroscopy-assisted endoscopic retrograde cholangiopancreatography (ERCP) in Roux-en-Y gastric bypass: a meta-analysis
}

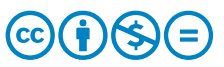

Authors

Fares Ayoub ${ }^{1}$, Tony S. Brar², Debdeep Banerjee ${ }^{3}$ Ali M. Abbas ${ }^{4}$, Yu Wang ${ }^{5}$, Dennis Yang², Peter V. Draganov²

Institutions

1 Section of Gastroenterology, Hepatology \& Nutrition, University of Chicago, Illinois, United States

2 Division of Gastroenterology, Hepatology and Nutrition, University of Florida, Gainesville, Florida, United States

3 Department of Medicine, University of Florida, Gainesville, Florida, United States

4 Brigham and Women's Hospital, Division of Gastroenterology, Harvard Medical School, Boston, Massachusetts, United States

5 Department of Biostatistics, University of Florida, Gainesville, Florida, United States

submitted 10.7.2019

accepted after revision $\quad 30.10 .2019$

Bibliography

DOI https://doi.org/10.1055/a-1070-9132 |

Endoscopy International Open 2020; 08: E423-E436

(c) Georg Thieme Verlag KG Stuttgart · New York eISSN 2196-9736

Corresponding author

Peter V. Draganov, MD, Professor of Medicine, Division of Gastroenterology, Hepatology \& Nutrition, University of Florida, 1329 SW 16th Street, Gainesville, FL 32608

Fax: +1-352-627-9002

peter.draganov@medicine.ufl.edu

Supplementary material

Online content viewable at:

https://doi.org/10.1055/a-1070-9132

\section{ABSTRACT}

Background and study aims Endoscopic retrograde cholangiopancreatography (ERCP) is technically challenging in patients with Roux-en-Y gastric bypass (RYGB) anatomy, which is increasing in frequency given the rise of obesity. Laparoscopy-assisted ERCP (LA-ERCP) and enteroscopy-assisted ERCP (EA-ERCP) are distinct approaches with their respective strengths and weaknesses. We conducted a metaanalysis comparing the procedural time, rates of success and adverse events of each method.

Patients and methods A search of PubMed, EMBASE and the Cochrane library was performed from inception to October 2018 for studies reporting outcomes of LA or EAERCP in patients with RYGB anatomy. Studies using single, double, 'short' double-balloon or spiral enteroscopy were included in the EA-ERCP arm. Outcomes of interest included procedural time, papilla identification, papilla cannulation, therapeutic success and adverse events. Therapeutic success was defined as successful completion of the originally intended diagnostic or therapeutic indication for ERCP.

Results A total of 3859 studies were initially identified using our search strategy, of which 26 studies met the inclusion criteria. The pooled rate of therapeutic success was significantly higher in LA-ERCP $(97.9 \%$; $95 \% \mathrm{Cl}$ : $96.7-$ $98.7 \%)$ with little heterogeneity $\left(I^{2}=0.0 \%\right)$ when compared to EA-ERCP (73.2\%; 95\% Cl: 62.5-82.6\%) with significant heterogeneity $\left(I^{2}: 80.2 \%\right)$. Conversely, the pooled rate of adverse events was significantly higher in LA-ERCP (19.0\%; $95 \% \mathrm{Cl}: 12.6-26.4 \%)$ when compared to EA-ERCP (6.5\%; $95 \% \mathrm{Cl}: 3.9-9.6 \%)$. The pooled mean procedure time for LA-ERCP was 158.4 minutes (SD \pm 20 ) which was also higher than the mean pooled procedure time for EA-ERCP at 100.5 minutes (SD \pm 19.2).

Conclusions LA-ERCP is significantly more effective than EA-ERCP in patients with RYGB but is associated with a higher rate of adverse events and longer procedural time. 


\section{Introduction}

The obesity epidemic continues to rise, with a doubling of global prevalence of obesity from $6.4 \%$ in 1980 to $12 \%$ in 2008 driven by rising incidence in Asia [1]. Bariatric surgery has been shown to be more effective for weight loss than medical therapy, with Roux-en-Y gastric bypass (RYGB) being considered the standard of care over the past decade. While the rapid weight loss experienced after bariatric surgery is desirable, it has been associated with changes in the composition of bile and the subsequent development of gallstones [2]. This invariably leads to a proportion of patients developing choledocholithiasis with complications ranging from asymptomatic elevations in liver enzymes to biliary pancreatitis [3]. When such complications arise, endoscopic retrograde cholangiopancreatography $(E R C P)$ is often indicated.

Performance of ERCP in RYGB patients can be technically challenging for several reasons. The Roux limb is intentionally created long to promote weight loss and typically exceeds 100 $\mathrm{cm}$ in length making the distance traversed by the endoscope significantly longer than standard ERCP [4]. Furthermore, the native papilla is more challenging to cannulate as compared to surgical bilio-enteric anastomosis due to the "upside-down" configuration and limited availability of accessory instruments that are designed for long endoscopes [5]. Thus, the combination of the long enteral limb and native papilla in RYGB makes for the most challenging ERCP of all post-surgical configurations. While several approaches exist, laparoscopy-assisted ERCP (LA-ERCP) and enteroscopy-assisted ERCP (EA-ERCP) are the most widely used modalities in RYGB patients [6].

LA-ERCP is performed by laparoscopically creating a gastrostomy through which a standard duodenoscope can be advanced into the excluded stomach and duodenum [7]. Studies have shown this method to have high rates of success, however, it is resource intensive and presents several technical risks and challenges [7]. This includes the logistical difficulties of coordinating surgeon, anesthetist and gastroenterologist schedules [8] as well as a higher overall adverse event (AE) rate than standard ERCP due to the laparoscopic approach [9].

EA-ERCP is performed utilizing overtube-based (single, double balloon or spiral) enteroscopy where a special endoscope is passed orally through the Roux limb and the jejunostomy up to the pancreaticobiliary limb to identify the papilla [10]. EA-ERCP has its limitations as well, including tortuosity of the endoscope trajectory, unstable working platform, suboptimal accessory performance due to the small diameter of the working channel and tangential view of the papilla [11].

While both LA-ERCP and EA-ERCP are considered safe and are widely used, their actual success and $A E$ rates have varied across studies. We conducted a meta-analysis comparing success rates, procedural time and AEs of LA-ERCP and EA-ERCP in patients status post RYGB with a native papilla.

\section{Patients and methods}

This meta-analysis was registered with the University Of York International Prospective Register Of Systematic Reviews (PROSPERO, Registration number CRD42018114884). This study was performed in accordance with the criteria established in the Preferred Reporting Items for Systematic Reviews and Meta-Analyses (PRISMA) guidelines.

\section{Search strategy and study selection}

Studies were identified by performing a literature search of three electronic databases (MEDLINE through PubMed, EMBASE and the Cochrane Library) with the last search performed in October 2018. The detailed search strategy is outlined in supplementary - Table 1 . We attempted to identify additional studies by reviewing the reference list of all included studies and manual search to retrieve other relevant articles that may have been missed on the initial search strategy. Three investigators (F.A. and T.B. and D.B.) screened all titles and abstracts for relevance to the study. The full text of potentially eligible studies was subsequently reviewed by the three investigators (F.A. and T.B. and D.B.). Disagreements were resolved by consensus or by consulting with a third investigator (P.V.D.).

\section{Inclusion and exclusion criteria}

Inclusion criteria were: (1) retrospective or prospective, case series, case-control, or cohort studies and clinical trials (including randomized clinical trials); (2) studies involving patients who are status post RYGB requiring ERCP utilizing either a LA or EA approach (single, double, "short” double balloon or spiral enteroscopy) (3) studies reporting papilla identification rate, cannulation rate, therapeutic/diagnostic success and procedural adverse events. Exclusion criteria were: (1) conference abstracts, case reports and case series with less than 5 patients, (2) studies in languages other than English (3) studies only involving patients with non-RYGB configurations (4) reviews, commentaries, surveys, (5) and duplicate studies.

\section{Data extraction}

Data from each eligible study were extracted using a standardized data extraction sheet. The extracted data included: (1) study authors, (2) year of publication, (3) setting (location), (4) study period, (5) patient demographics (age, gender), (6) number of patients/procedures, (7) indications for ERCP (8) ERCP approach (LA or EA) (9) papilla identification rate, cannulation rate, therapeutic/diagnostic success rate, (10) procedural adverse events and (11) procedural time.

\section{Outcomes and definitions}

The primary aim of this study was to conduct a meta-analysis comparing the papilla identification rate, cannulation rate, therapeutic/diagnostic success rate of $L A$ versus EA-ERCP in patients who are status post RYGB with a native papilla. A secondary aim was to compare the adverse event rates and procedural time associated with each modality. Papilla identification was defined as successful visualization of the papilla of Vater using the endoscope. Successful cannulation was defined as success- 
- Table1 Study characteristics for the enteroscopy-assisted endoscopic retrograde cholangiopancreatography arm.

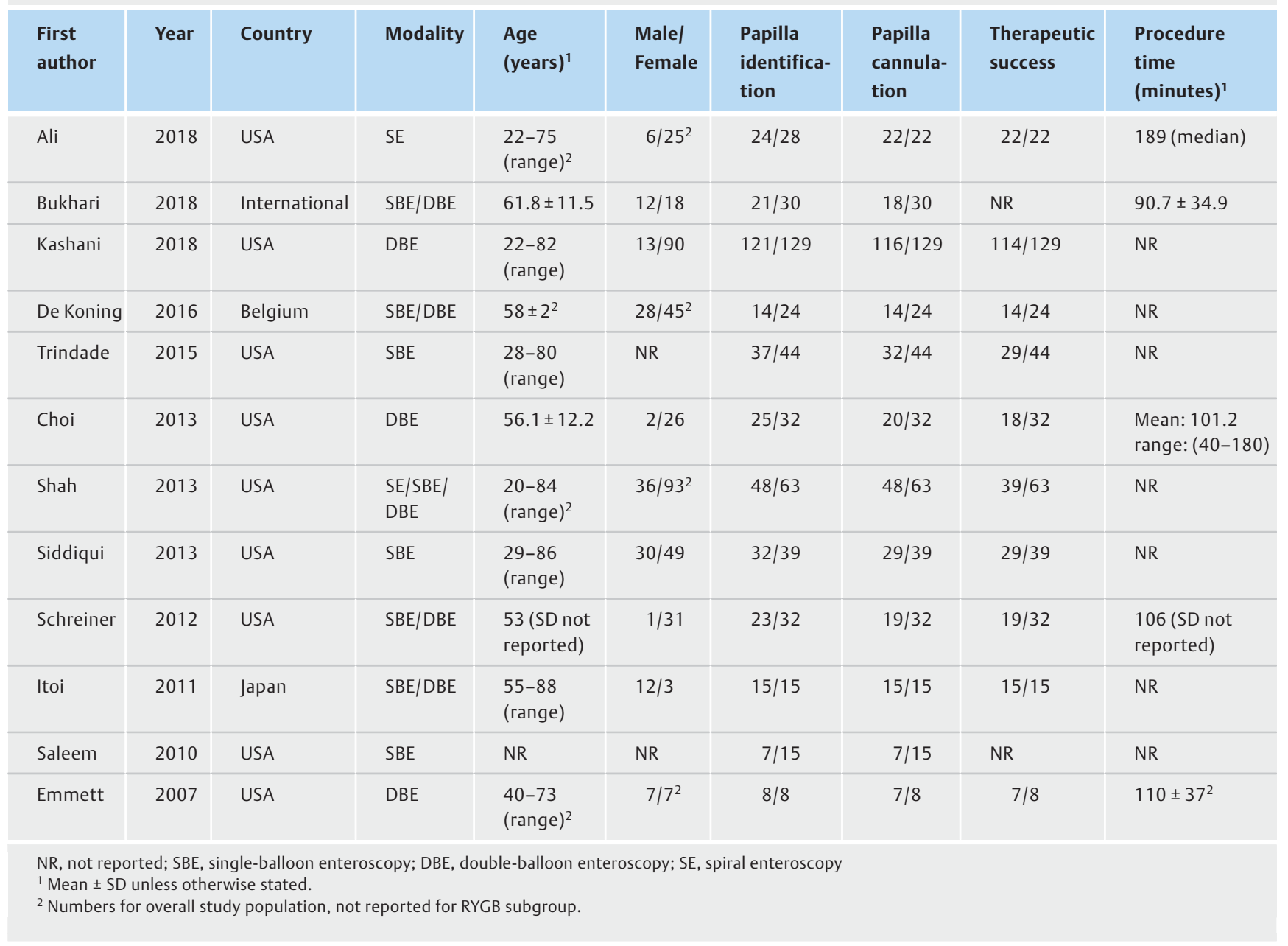

ful introduction of a catheter into the desired duct. Therapeutic/diagnostic success was defined as successful completion of the originally intended diagnostic or therapeutic indication for ERCP as clinically indicated.

\section{Assessment of methodologic quality}

The quality of studies was assessed using the Newcastle Ottawa scale (NOS) [12]. Because the majority of included studies were case series, we utilized a modified version of the NOS appropriate for our analysis. This tool removes from the NOS the items that relate to comparability between two arms and retains items that assess representation and selection of cases as well as ascertainment of exposure and outcome. A point is assigned to each component of the modified scale, with the highest possible score being 6/6. Studies were considered to be high quality if they scored $6 / 6$, moderate quality if they scored $5 / 6$ and low quality if they scored $4 / 6$ or less. The quality of all studies was assessed by three investigators (F.A, T.B., D.B.). Egger's regression test was used to assess for publication bias.

\section{Statistical analysis}

Pooled rates were calculated utilizing a random effects model and the Freeman-Tukey arcsine transformation was used [13]. The Cochran $\mathrm{Q}$ test and $\mathrm{I}^{2}$ were used to assess heterogeneity of included studies. $\mathrm{I}^{2}$ values $<25 \%, 25 \%$ to $50 \%$ and $>50 \%$ were considered to represent low, moderate, and high heterogeneity, respectively. $P<0.05$ was considered significant and all tests were two-tailed. The study was performed in accordance with the PRISMA recommendations for reporting systematic reviews and meta-analyses. Analysis was conducted using Stata, version 15 (Stata Corp, College Station, Texas, United States).

\section{Results}

\section{Search results}

The flow diagram for study selection is depicted in > Fig.1. Overall, 3859 studies were identified using our search strategy, of which 1615 were duplicates. Of the remaining 2244 studies after duplicate removal, 2134 were excluded after screening titles and abstracts. Full text review was then performed on 110 studies using the predefined inclusion and exclusion criteria, after which 26 studies were retained. Twenty two were case series (1 prospective, 21 retrospective) [5, $8,10,14-31]$, two 


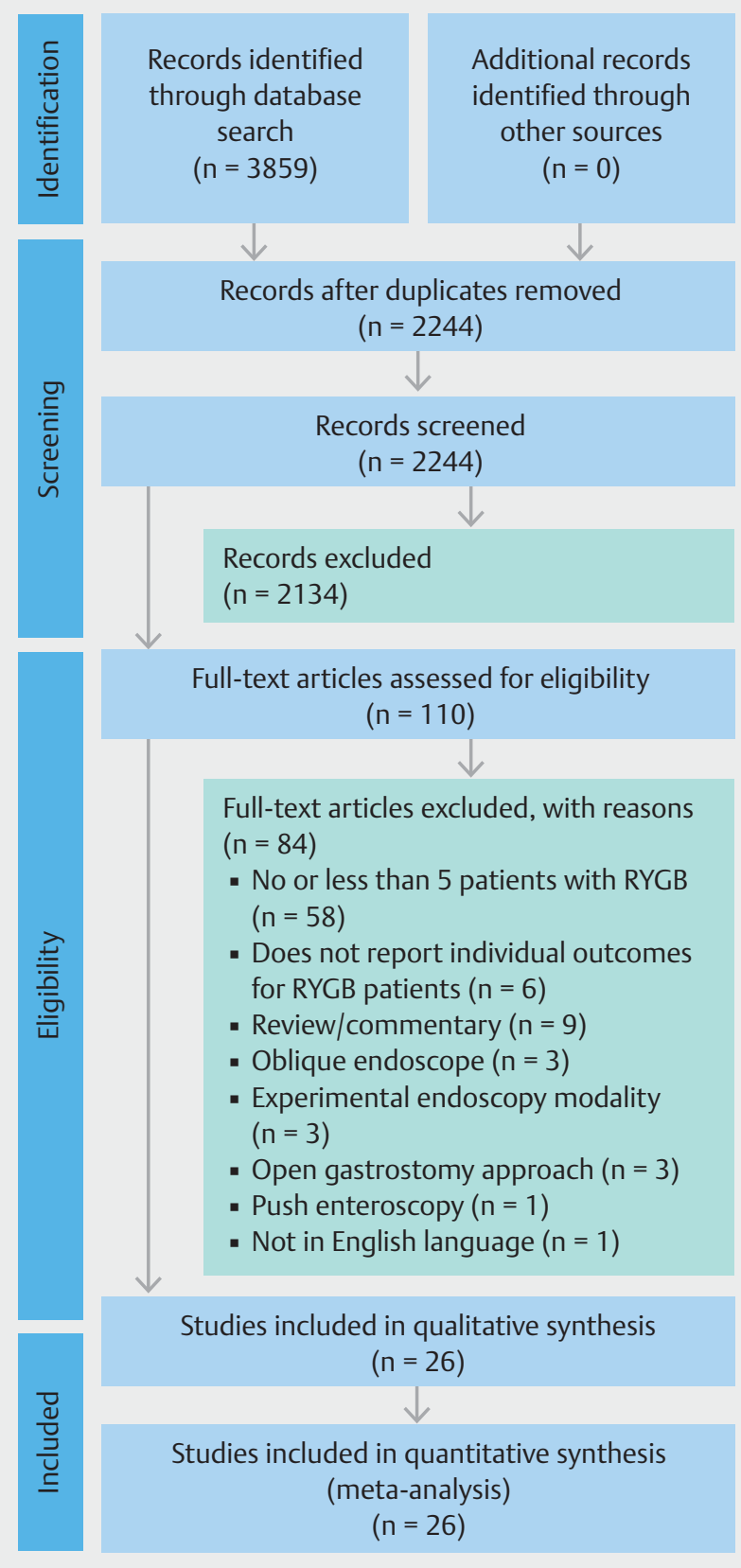

- Fig. 1 PRISMA flow diagram. From: Moher D, Liberati A, Tetzlaff J, Altman DG, The PRISMA Group (2009). Preferred Reporting Items for Systematic Reviews and Meta-Analyses: The PRISMA Statement. PLoS Med 6(7): e1000097. doi:10.1371/journal.pmed1000097

were retrospective cohort studies comparing balloon EA to LAERCP $[6,32]$, and two were retrospective cohort studies comparing EA or LA-ERCP to other approaches where data on laparoscopy or balloon enteroscopy was extracted and used for the pooled analysis [33, 34]. Studies were published between 2007 and 2018. Eight studies were multi-center studies, two of which were conducted internationally. Eighteen studies were conducted in the United States, four in Europe, one in Brazil, one in Japan. Data from the laparoscopy arm were not used from two cohort studies [6,32] because the same data were included in the multicenter study by Abbas et al [8].

\section{Patient population and study characteristics}

A total of 427 patients underwent 459 EA-ERCPs, and 882 patients underwent 886 LA-ERCPs. Study characteristics are summarized in > Table 1 and $>$ Table 2.

\section{Indications and adverse events}

Detailed data on procedural indications were reported in nine of 12 EA-ERCP studies and in 13 of 14 LA-ERCP studies. The most common procedural indication in the LA-ERCP arm was choledocholithiasis in $48 \%$ of cases (408/847), compared to $74 \%(280 / 380)$ in EA-ERCP. Adverse events were reported by $10 / 12$ in the EA-ERCP arm and all studies in the LA-ERCP arm. In the EA-ERCP arm, the most commonly reported AE was pancreatitis in $5 \%(23 / 459)$ of cases. Only one study [27] described the severity of pancreatitis where one of five pancreatitis cases was considered to be severe. Small bowel perforation was uncommon and was reported in $1 \%(6 / 459)$ of cases. Death was rare with only one case reported by Shah et al. [27] in the EAERCP arm where a patient developed an embolic stroke postprocedurally and decision was made to withdraw care.

In the LA-ERCP arm, 12 of 14 studies classified AEs into either ERCP or laparoscopy-related. The most common ERCPrelated $A E$ was pancreatitis, reported in $6 \%(53 / 847)$ of cases. Perforation was again uncommon and was reported in $1 \%$ (10/ 847 ) of cases. The most common laparoscopy-related adverse event was infection, reported in $5 \%(44 / 847)$ of cases, the majority of which were localized in nature. There were no reports of death, however there was one reported case of tension pneumothorax in the study by Lopes et al. [23] which was caused by an indwelling percutaneous transhepatic cholangiogram (PTC) catheter crossing the diaphragm, however this was promptly recognized and managed with chest tube insertion. - Table 3 and $>$ Table 4 summarize the indications and AEs for both EA and LA-ERCP.

\section{Quality assessment}

Risk of bias in the 26 studies was evaluated according to the modified Newcastle-Ottawa assessment scale and is shown in Supplementary Table 2. Overall, 20 of 26 studies (77\%) were found to be of moderate to high quality and six of 26 studies $(33 \%)$ were found to be low quality. Most quality issues were related to a lack of adequate description of the characteristics and outcomes of the RYGB cohort in studies that included patients with a broad variety of post-surgical anatomy. It is important to note that majority of included studies were retrospective case series, which inherently affects overall study quality.

\section{Meta-analysis results}

The pooled results of papilla identification, papilla cannulation and therapeutic success rates are summarized in $>$ Table 5. 
- Table2 Study characteristics for laparoscopy-assisted endoscopic retrograde cholangiopancreatography arm.

\begin{tabular}{|c|c|c|c|c|c|c|c|c|}
\hline First author & Year & Country & Age (years) ${ }^{1}$ & $\begin{array}{l}\text { Male/ } \\
\text { Female }\end{array}$ & $\begin{array}{l}\text { Papilla } \\
\text { identifica- } \\
\text { tion }\end{array}$ & $\begin{array}{l}\text { Papilla } \\
\text { cannula- } \\
\text { tion }\end{array}$ & $\begin{array}{l}\text { Therapeutic } \\
\text { success }\end{array}$ & $\begin{array}{l}\text { Procedure time } \\
\text { (minutes) })^{1,2}\end{array}$ \\
\hline Abbas & 2018 & $\begin{array}{l}\text { Interna- } \\
\text { tional }\end{array}$ & 51 (IQR, 43-61) & $91 / 488$ & $573 / 579$ & $567 / 579$ & $567 / 579$ & $\begin{array}{l}152 \text { minutes } \\
(\mathrm{IQR}, 109-210)\end{array}$ \\
\hline Kedia & 2018 & USA & $55(33-80)$ & $7 / 36$ & $42 / 43$ & $42 / 43$ & $42 / 43$ & 184 \\
\hline Yancey & 2018 & USA & $55.8(29-67)$ & NR & $15 / 16$ & $15 / 16$ & $15 / 16$ & NR \\
\hline Frederiksen & 2017 & Denmark & Median: $46(25-65)$ & $4 / 24$ & $31 / 31$ & $31 / 31$ & $31 / 31$ & NR \\
\hline Lim & 2017 & USA & $50.3 \pm 9.8$ & $0 / 35$ & $35 / 35$ & $35 / 35$ & $35 / 35$ & NR \\
\hline Bowman & 2016 & USA & $48.5(25-71)$ & $4 / 11$ & $16 / 16$ & $16 / 16$ & $16 / 16$ & NR \\
\hline Paranandi & 2016 & UK & Median: 44 & $0 / 7$ & $7 / 7$ & $7 / 7$ & $7 / 7$ & 96 \\
\hline Grimes & 2015 & USA & $48.5(23-69)$ & $36 / 2$ & $36 / 38$ & $36 / 38$ & NR & 265 \\
\hline Snauwaert & 2015 & Belgium & Median: 57 (26-79) & $5 / 18$ & $23 / 23$ & $23 / 23$ & $23 / 23$ & NR \\
\hline Falcao & 2012 & Brazil & $35.3(27-52)$ & $4 / 19$ & $23 / 23$ & $23 / 23$ & $23 / 23$ & 92.69 \\
\hline Saleem & 2012 & USA & $51 \pm 12.58(25-70)$ & $3 / 12$ & $15 / 15$ & $15 / 15$ & $15 / 15$ & 45 \\
\hline Bertin & 2011 & USA & NR & NR & $22 / 22$ & $22 / 22$ & NR & 236 \\
\hline Gutierrez & 2009 & USA & $46(27-72)$ & $4 / 24$ & $28 / 28$ & $28 / 28$ & NR & 200 \\
\hline Lopes & 2009 & USA & $40(19-55)$ & $1 / 9$ & $9 / 10$ & $9 / 10$ & $9 / 10$ & 89 \\
\hline \multicolumn{9}{|c|}{$\begin{array}{l}\text { NR, not reported } \\
{ }^{1} \text { Mean } \pm \text { SD, (rang } \\
{ }^{2} \text { Numbers for ove }\end{array}$} \\
\hline
\end{tabular}

\section{Papilla identification}

All studies in the EA arm and the LA arm reported papilla identification rates ( $\mathbf{F i g . 2}$, $>$ Fig. $\mathbf{3}$ ). The pooled rate of papilla identification in LA-ERCP was $98.5 \%$ (95\% confidence interval [Cl]: 97.6-99.2\%) with no heterogeneity identified in the pooled analysis $\left(I^{2}=0.0 \%\right)$. This was higher than the pooled rate of papilla identification in EA-ERCP at $80.0 \%(95 \% \mathrm{Cl}$ : $71.3-87.4 \%$ ) with studies demonstrating a high degree of heterogeneity (I2: $77.5 \%)$. Among the EA-ERCP studies, four reported papilla identification rates utilizing single-balloon enteroscopy with a pooled rate of 78.5\% (95\% Cl: 56.6-94.1\%), 3 studies reported papilla identification rates utilizing doubleballoon enteroscopy with a pooled rate of $80.4 \%(95 \% \mathrm{Cl}$ : 71.6-88.0\%) and 2 studies reported papilla identification rates utilizing spiral enteroscopy with a pooled rate of $78.9 \%(95 \% \mathrm{Cl}$ : $65.8-89.5 \%)$. There was no evidence of substantial publication bias based on visual inspection of the funnel plot and Egger's regression test (Supplementary Fig. 1a, 1b).

\section{Papilla cannulation}

All studies in the EA-ERCP arm and the LA-ERCP arm reported papilla cannulation rates ( $>$ Fig. 4 , $>$ Fig. 5). The pooled rate of papilla cannulation LA-ERCP was $97.8 \%$ (95\% confidence interval [Cl]: 96.7-98.7\%) with no heterogeneity identified in the pooled analysis $\left(I^{2}=0.0 \%\right)$. This was higher than the pooled rate of papilla cannulation in EA-ERCP at $73.0 \%$ (95\% Cl: $63.6-$ $81.5 \%$ ) with studies demonstrating a high degree of heteroge- neity (I2: 77.4\%). Among EA-ERCP studies, four reported papilla cannulation rates utilizing single-balloon enteroscopy with a pooled rate of $75.3 \%$ (95\% Cl: 53.4-91.9\%), three studies reported papilla identification rates utilizing double-balloon enteroscopy with a pooled rate of $72.3 \%(95 \% \mathrm{Cl}$ : $60.0-83.1 \%)$ and two studies reported papilla cannulation rates utilizing spiral enteroscopy with a pooled rate of $89.4 \%$ (95\% Cl: $51.3-$ $98.8 \%$ ). There was no evidence of substantial publication bias based on visual inspection of the funnel plot and Egger's regression test (Supplementary Fig.2a, Supplementary Fig. 2b).

\section{Therapeutic success}

Ten studies in the EA-ERCP arm and 11 studies in the LA-ERCP arm reported therapeutic success rates ( $>$ Fig. 6, $>$ Fig. 7). The pooled rate of therapeutic success in LA-ERCP was $97.9 \%$ (95\% confidence interval $[\mathrm{CI}]$ : 96.7-98.7\%) with no heterogeneity identified in the pooled analysis $\left(I^{2}=0.0 \%\right)$. This was higher than the pooled rate of therapeutic success in EA-ERCP at 73.2\% (95\% Cl: 62.5-82.6\%) with studies demonstrating a high degree of heterogeneity $\left(\mathrm{I}^{2}\right.$ : $\left.80.2 \%\right)$. Among EA-ERCP studies, three studies reported therapeutic success rates utilizing single-balloon enteroscopy with a pooled rate of $77.2 \%$ (95\% Cl: 48.9-96.1\%), three studies reported therapeutic success rates utilizing double-balloon enteroscopy with a pooled rate of $65.8 \%$ (95\% Cl: 54.2-76.5\%) and two studies reported therapeutic success rates utilizing spiral enteroscopy with a 
Table 3 Complications and procedural indications for enteroscopy-assisted endoscopic retrograde cholangiopancreatography arm.

\begin{tabular}{|c|c|c|c|}
\hline First author & Year & Indications (n) & Complications (n) \\
\hline Ali & 2018 & $\begin{array}{l}\text { Choledocholithiasis (14) } \\
\text { Biliary stricture (8) } \\
\text { Sphincter of Oddi dysfunction (5) } \\
\text { Stent placement/removal (4) } \\
\text { Pancreatitis (1) } \\
\text { Type III choledochocele (1) } \\
\text { Bile leak (1) }\end{array}$ & None \\
\hline Bukhari & 2018 & $\begin{array}{l}\text { Choledocholithiasis ( } 30 \text { ) } \\
\text { Benign biliary stricture (5) } \\
\text { Sphincter of Oddi dysfunction (2) } \\
\text { Cholangitis (2) }\end{array}$ & $\begin{array}{l}\text { Pancreatitis ( } 1 \text { ) } \\
\text { Cholangitis ( } 1 \text { ) } \\
\text { Small bowel perforation ( } 1 \text { ) }\end{array}$ \\
\hline Kashani & 2018 & $\begin{array}{l}\text { Sphincter of Oddi dysfunction (66) } \\
\text { Choledocholithiasis (26) } \\
\text { Pancreatitis (9) } \\
\text { Biliary stricture (8) } \\
\text { Bile leak (8) } \\
\text { Cholangitis (6) } \\
\text { Abnormal liver tests (5) } \\
\text { Recurrent liver abscess (1) }\end{array}$ & $\begin{array}{l}\text { Pancreatitis (10) } \\
\text { Small bowel perforation ( } 2 \text { ) } \\
\text { Cholangitis ( } 1 \text { ) }\end{array}$ \\
\hline De Koning & 2016 & NR & NR \\
\hline Trindade & 2015 & $\begin{array}{l}\text { Choledocholithiasis (29) } \\
\text { Cholangitis (10) } \\
\text { Abnormal liver tests (9) } \\
\text { Benign biliary stricture (4) } \\
\text { Bile leak (4) }\end{array}$ & Pancreatitis (3) \\
\hline Choi & 2013 & $\begin{array}{l}\text { Choledocholithiasis (16) } \\
\text { Sphincter of Oddi dysfunction (6) } \\
\text { Biliary stricture (4) } \\
\text { Bile leak (2) }\end{array}$ & Pancreatitis (1) \\
\hline Shah & 2013 & $\begin{array}{l}\text { Abnormal liver enzymes + dilated bile ducts (62) } \\
\text { Dilated bile ducts on non-invasive imaging (21) } \\
\text { Cholangitis (20) } \\
\text { Abnormal liver enzymes (11) } \\
\text { Pancreatitis (8) } \\
\text { Other (7) }\end{array}$ & $\begin{array}{l}\text { Mild pancreatitis (4) } \\
\text { Severe pancreatitis (1) } \\
\text { Bleeding (1) } \\
\text { Abdominal pain leading to re-admission ( } 3 \text { ) } \\
\text { Throat pain requiring physician contact (4) } \\
\text { Perforation (2) } \\
\text { Death (1) }\end{array}$ \\
\hline Siddiqui & 2013 & $\begin{array}{l}\text { Choledocholithiasis (48) } \\
\text { Biliary stricture (18) } \\
\text { Stent removal (5), } \\
\text { Sphincter of Oddi dysfunction (3), } \\
\text { Bile leak (3) } \\
\text { Pancreatic stricture (2) }\end{array}$ & $\begin{array}{l}\text { Abdominal pain ( } 3 \text { ) } \\
\text { Pancreatitis ( } 3 \text { ) } \\
\text { Post-procedural bleeding ( } 1 \text { ) }\end{array}$ \\
\hline Schreiner & 2012 & $\begin{array}{l}\text { "Preprocedure indications for ERCP included (1) dilation of the } \\
\text { pancreaticobiliary tree in the setting of laboratory abnormalities } \\
\text { or clinical symptoms; (2) stones seen on imaging; and/or ( } 3 \text { ) ab- } \\
\text { dominal pain with abnormal laboratory test results suggesting } \\
\text { biliopancreatic origin." }\end{array}$ & Pancreatitis (1) \\
\hline Itoi & 2011 & Choledocholithiasis (15) & None \\
\hline Saleem & 2010 & $\begin{array}{l}\text { "Cholestasis, acute cholangitis, recurrent primary sclerosing } \\
\text { cholangitis with strictures, and choledocholithiasis." }\end{array}$ & None \\
\hline Emmett & 2007 & $\begin{array}{l}\text { Repeat procedure (6) } \\
\text { Recurrent pancreaticobiliary pain (5) } \\
\text { Abnormal liver tests (4) } \\
\text { Cholangitis (2) } \\
\text { Chronic pancreatitis (2) } \\
\text { Acute pancreatitis (1) }\end{array}$ & None \\
\hline
\end{tabular}

Complications and indications reported for overall study population when data on specific RYGB patients are not reported in individual studies. NR, not reported. 
- Table4 Complications and procedural indications for the laparoscopy-assisted assisted endoscopic retrograde cholangiopancreatography arm.

\begin{tabular}{|c|c|c|c|c|}
\hline First author & Year & Indications (n) & Complications (n) & $\begin{array}{l}\text { Conversion to } \\
\text { open (n) }\end{array}$ \\
\hline Abbas & 2018 & $\begin{array}{l}\text { Choledocholithiasis (254) } \\
\text { Papillary stenosis (102) } \\
\text { Dilated duct (75) } \\
\text { Pancreatitis (56) } \\
\text { Abnormal liver function tests (46) } \\
\text { Bile duct stricture (20) } \\
\text { Post cholecystectomy pain (10) } \\
\text { Abdominal pain (9) } \\
\text { Bile leak (7) } \\
\text { Ampullary lesion (7) } \\
\text { Biliary stent removal (3) } \\
\text { Dilated pancreatic duct (3) } \\
\text { Abnormal intraoperative cholangiogram (2) } \\
\text { Pancreatic duct stone (1) }\end{array}$ & $\begin{array}{l}\text { Laparoscopy-related } \\
\text { Other postoperative infections (24) } \\
\text { Laparoscopy-related bleeding (10) } \\
\text { Gastric site leak (7) } \\
\text { Gastric tube site infection (7) } \\
\text { Postoperative respiratory adverse events (6) } \\
\text { Postoperative cardiovascular adverse events (4) } \\
\text { Laparoscopy-related perforation (3) } \\
\text { Other laparoscopic related (11) } \\
\text { ERCP-related } \\
\text { Pancreatitis (43) } \\
\text { Cholangitis (6) } \\
\text { ERCP-related bleeding (3) } \\
\text { ERCP-related perforation (2) } \\
\text { Stent migration (1) }\end{array}$ & 29 \\
\hline Kedia & 2018 & $\begin{array}{l}\text { Choledocholithiasis (54) } \\
\text { Papillary stenosis (5) }\end{array}$ & $\begin{array}{l}\text { ERCP-related } \\
\text { Perforation (2) } \\
\text { Laparoscopy-related } \\
\text { Intraperitoneal abscess (2) } \\
\text { Wound dehiscence (1) } \\
\text { Bleeding (1) } \\
\text { Abdominal wall seroma (1) } \\
\text { Cellulitis (1) }\end{array}$ & 4 \\
\hline Yancey & 2018 & $\begin{array}{l}\text { "Choledocholithiasis, cholangitis, and radio- } \\
\text { graphic or clinical evidence of common bile } \\
\text { duct (CBD) obstruction." }\end{array}$ & $\begin{array}{l}\text { ERCP-related } \\
\text { Necrotizing pancreatitis (1) } \\
\text { Laparoscopy-related } \\
\text { None }\end{array}$ & 1 \\
\hline Frederiksen & 2017 & Choledocholithiasis (31) & $\begin{array}{l}\text { ERCP-related } \\
\text { Perforation (2) } \\
\text { Pancreatitis (2) } \\
\text { Laparoscopy-related } \\
\text { Intraperitoneal abscess (3) } \\
\text { Abdominal hematoma (3) } \\
\text { Wound dehiscence (1) }\end{array}$ & 2 \\
\hline Lim & 2017 & $\begin{array}{l}\text { Sphincter of Oddi dysfunction (35) } \\
\text { Choledocholithiasis (10) } \\
\text { Biliary stricture (2) } \\
\text { Pseudocyst (1) } \\
\text { Cystic duct leak (1) } \\
\text { Pancreatic leak (1) }\end{array}$ & $\begin{array}{l}\text { ERCP-related } \\
\text { Pancreatitis (3) } \\
\text { Laparoscopy-related } \\
\text { None }\end{array}$ & NR \\
\hline Bowman & 2016 & $\begin{array}{l}\text { Choledocholithiasis (5) } \\
\text { Recurrent pancreatitis (3) } \\
\text { Ampullary mass ( } 1 \text { ) } \\
\text { Sphincter of Oddi dysfunction (1) } \\
\text { Biliary stricture (1) }\end{array}$ & $\begin{array}{l}\text { ERCP-related } \\
\text { None } \\
\text { Laparoscopy-related } \\
\text { Abdominal abscess (1) } \\
\text { Incisional hernia (1) } \\
\text { Wound dehiscence (1) }\end{array}$ & 1 \\
\hline Paranandi & 2016 & $\begin{array}{l}\text { Choledocholithiasis (5) } \\
\text { Papillary fibrosis (1) } \\
\text { Retained biliary stent (1) }\end{array}$ & $\begin{array}{l}\text { ERCP-related } \\
\text { Pancreatitis (1) } \\
\text { Laparoscopy-related } \\
\text { Port-site infection (1) }\end{array}$ & 0 \\
\hline
\end{tabular}


Table 4 (Continuation)

\begin{tabular}{|c|c|c|c|c|}
\hline First author & Year & Indications (n) & Complications (n) & $\begin{array}{l}\text { Conversion to } \\
\text { open (n) }\end{array}$ \\
\hline Grimes & 2015 & $\begin{array}{l}\text { Chronic abdominal pain/sphincter of Oddi } \\
\text { dysfunction/pancreatic duct stenosis/chronic } \\
\text { pancreatitis ( } 80 \text { ) } \\
\text { Choledocholithiasis (5) }\end{array}$ & $\begin{array}{l}\text { ERCP-related } \\
\text { Duodenal perforation (2) } \\
\text { Laparoscopy related } \\
\text { G-tube site infection (4) } \\
\text { Posterior gastric wall injury (4) } \\
\text { Persistent gastro-cutaneous fistula (2) } \\
\text { Bleeding requiring transfusion (2) } \\
\text { Pneumoperitoneum (2) } \\
\text { Perforation (1) } \\
\text { Abdominal wall hematoma (1) }\end{array}$ & 1 \\
\hline Snauwaert & 2015 & $\begin{array}{l}\text { Choledocholithiasis (16) } \\
\text { Biliary pain (4) } \\
\text { Jaundice (3) }\end{array}$ & None & 2 \\
\hline Falcao & 2012 & $\begin{array}{l}\text { Choledocholithiasis (14) } \\
\text { Cholecystitis (6) } \\
\text { Obstructive jaundice (3) }\end{array}$ & $\begin{array}{l}\text { ERCP-related } \\
\text { Pancreatitis ( } 1 \text { ) } \\
\text { Laparoscopy related } \\
\text { None }\end{array}$ & 0 \\
\hline Saleem & 2012 & $\begin{array}{l}\text { Sphincter of Oddi dysfunction (9) } \\
\text { Choledocholithiasis (5) } \\
\text { Recurrent acute pancreatitis (1) }\end{array}$ & None & 0 \\
\hline Bertin & 2011 & $\begin{array}{l}\text { Sphincter of Oddi dysfunction (18) } \\
\text { Recurrent acute pancreatitis (4) }\end{array}$ & $\begin{array}{l}\text { ERCP-related } \\
\text { Perforation (1) } \\
\text { Laparoscopy related } \\
\text { Abdominal hematoma (1) } \\
\text { Bile leak (1) }\end{array}$ & 1 \\
\hline Gutierrez & 2009 & $\begin{array}{l}\text { Sphincter of Oddi dysfunction (13) } \\
\text { Pancreatitis (6) } \\
\text { Choledocholithiasis (5) } \\
\text { Cholangitis (3) } \\
\text { Pancreatic mass evaluation (2) } \\
\text { Gastrointestinal bleed (2) } \\
\text { Bile leak (1) }\end{array}$ & $\begin{array}{l}\text { ERCP-related } \\
\text { Perforation (1) } \\
\text { Laparoscopy-related } \\
\text { Gastrostomy site leak (2) } \\
\text { Gastrostomy site infection (1) }\end{array}$ & 1 \\
\hline Lopes & 2009 & $\begin{array}{l}\text { Choledocholithiasis (4) } \\
\text { Biliary stricture (3) } \\
\text { Sphincter of Oddi dysfunction (3) }\end{array}$ & $\begin{array}{l}\text { ERCP-related } \\
\text { Pancreatitis (2) } \\
\text { Laparoscopy-related } \\
\text { Tension pneumothorax (1) }\end{array}$ & 0 \\
\hline
\end{tabular}

Table 5 Summary of pooled outcomes for enteroscopy-assisted compared to laparoscopy-assisted endoscopic retrograde cholangiopancreatography.

\begin{tabular}{|c|c|c|c|c|c|c|}
\hline & \multicolumn{2}{|c|}{ Papilla identification } & \multicolumn{2}{|c|}{ Papilla cannulation } & \multicolumn{2}{|c|}{ Therapeutic success } \\
\hline & Pooled rate (\%) & $95 \% \mathrm{Cl}$ & Pooled rate (\%) & $95 \% \mathrm{Cl}$ & Pooled rate (\%) & $95 \% \mathrm{Cl}$ \\
\hline Enteroscopy-assisted ERCP & 80.0 & $71.3-87.4$ & 73.0 & $63.6-81.5$ & 73.2 & $62.5-82.6$ \\
\hline Single-balloon enteroscopy & 78.5 & $56.6-94.1$ & 75.3 & $53.4-91.9$ & 77.2 & 48.9-96.1 \\
\hline Double-balloon enteroscopy & 80.4 & $71.6-88.0$ & 72.3 & $60.0-83.1$ & 65.8 & $54.2-76.5$ \\
\hline Spiral enteroscopy & 78.9 & $65.8-89.5$ & 89.4 & $51.3-98.8$ & 85.5 & $34.1-97.3$ \\
\hline Laparoscopy-assisted ERCP & 98.5 & $97.6-99.2$ & 97.8 & $96.7-98.7$ & 97.9 & $96.7-98.7$ \\
\hline
\end{tabular}

$\mathrm{Cl}$, confidence interval; ERCP, endoscopic retrograde cholangiopancreatography. 


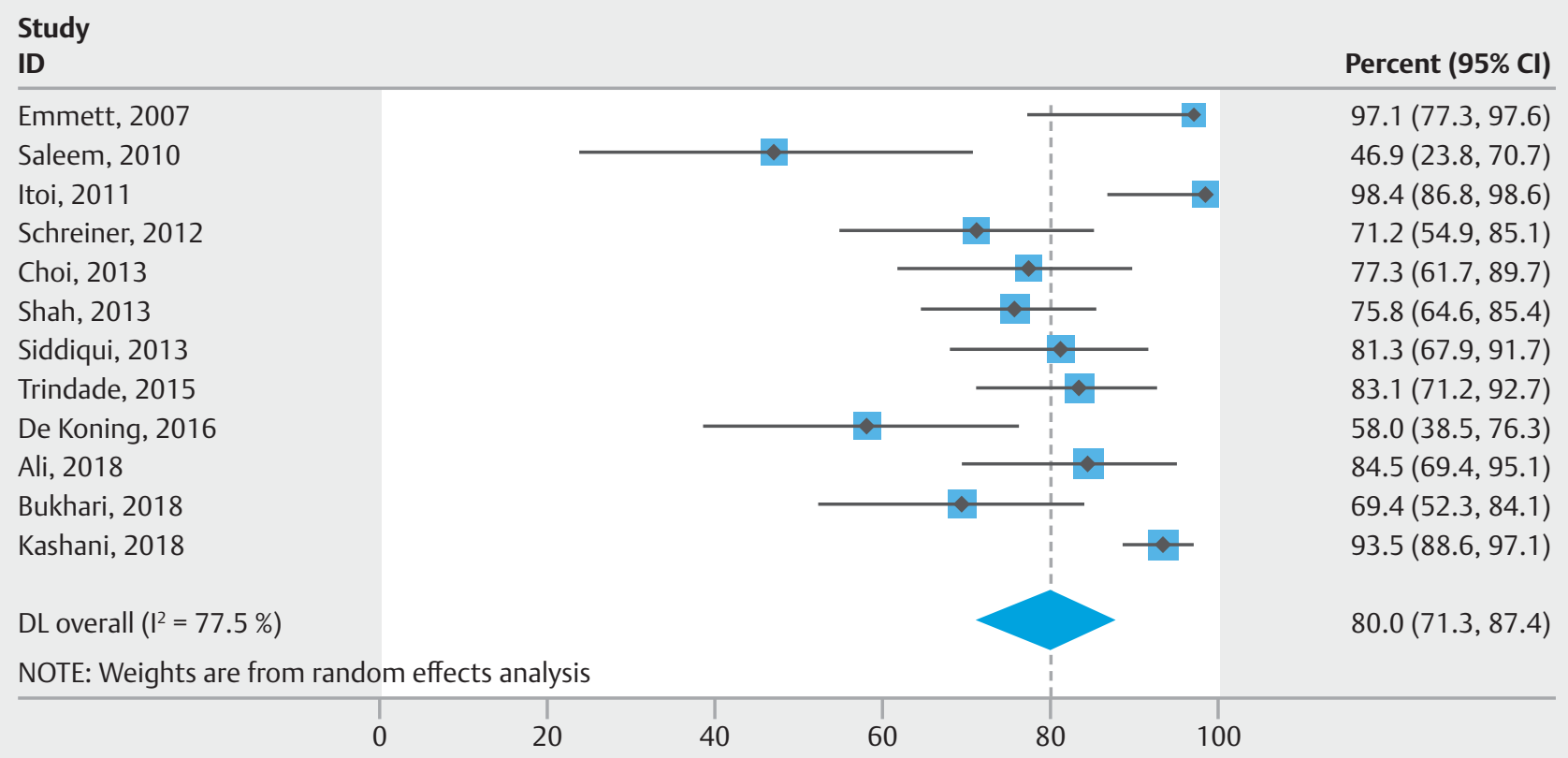

- Fig. 2 Pooled papilla identification rate of enteroscopy-assisted ERCP arm.

pooled rate of $85.5 \%(95 \% \mathrm{Cl}: 34.1-97.3 \%)$. There was no evidence of substantial publication bias based on visual inspection of the funnel plot and Egger's regression test (Supplementary Fig. 3a, Supplementary Fig.3b).

\section{Adverse events}

Ten of 12 studies in the EA-ERCP arm and all studies in the LAERCP arm reported post-procedural adverse events ( $\mathbf{F i g . 8}$, - Fig.9). Overall AE rates for the LA arm were calculated as a composite of ERCP-related adverse events, laparoscopy-related adverse events and conversion to open surgery. The pooled rate of overall AEs in LA-ERCP was $19.0 \%$ (95\% Cl: 12.6-26.4\%) with studies demonstrating a high degree of heterogeneity $\left(\mathrm{I}^{2}\right.$ : $74.1 \%)$. This was higher than the pooled rate of adverse events in EA-ERCP at $6.5 \%$ (95\% Cl: 3.9-9.6\%) with studies demonstrating low heterogeneity (I2: 16.2\%). Twelve of 14 LA-ERCP studies reported separate ERCP-related AEs, and the pooled ERCP-related $A E$ rate was $8 \%(95 \% \mathrm{Cl}: 5.4-10.9 \%)$ with low heterogeneity (I2: $15.6 \%)$. There was no evidence of substantial publication bias based on visual inspection of the funnel plot (Supplementary Fig.4a, Supplementary Fig.4b).

\section{Procedure duration}

Four of 12 studies in the EA arm and nine of 14 studies in the LA arm reported procedural time in minutes. Procedural time for the LA arm was calculated as a composite of laparoscopy and ERCP time since only one study reported separate laparoscopy and ERCP times. Pooled mean procedure time for LA-ERCP was 158.4 minutes ( $S D \pm 20$ ). This was higher than the mean pooled procedure time for EA-ERCP at 100.5 minutes (SD \pm 19.2 ),

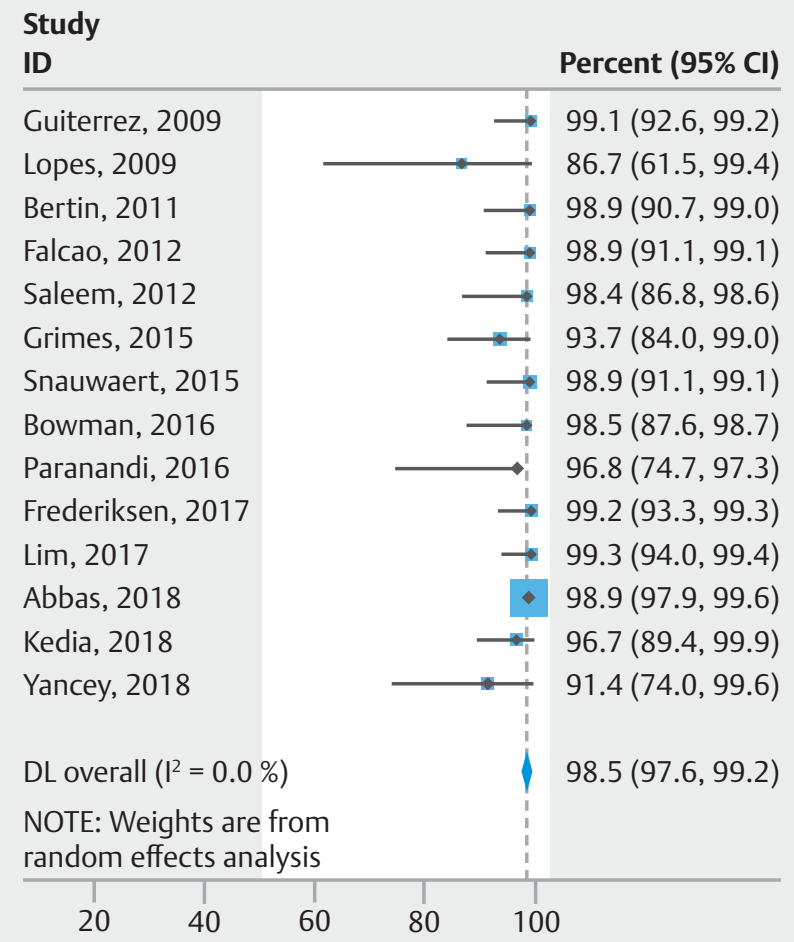

Fig. 3 Pooled papilla identification rate of laparoscopy-assisted ERCP arm. 


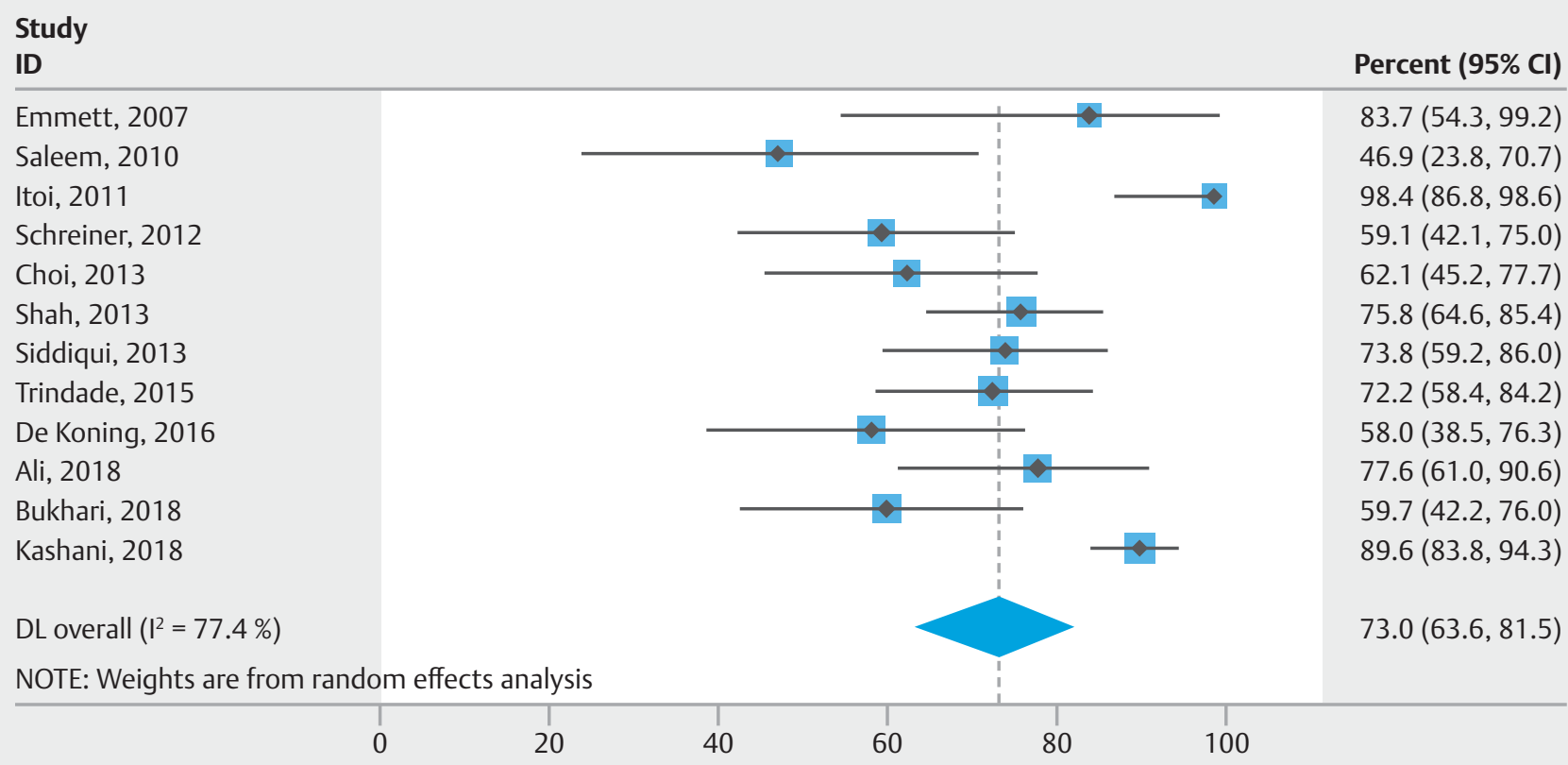

- Fig.4 Pooled papilla cannulation rate of enteroscopy-assisted ERCP arm.

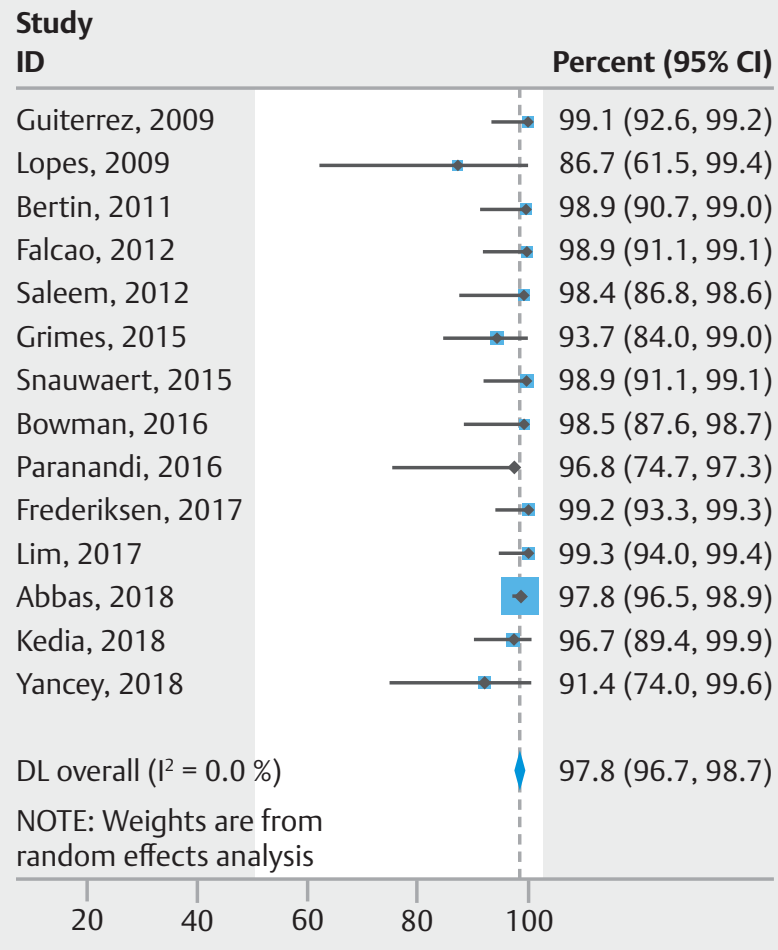

Fig. 5 Pooled papilla cannulation rate of laparoscopy-assisted ERCP arm.

\section{Discussion}

With the rise of the obesity epidemic and the popularity of bariatric surgery, patients with RYGB requiring ERCP are increasingly encountered in clinical practice. While several approaches exist, LA-ERCP and EA-ERCP are the most widely used modalities in RYGB patients [6]. LA-ERCP is performed by laparoscopically creating a gastrostomy through which a standard duodenoscope can be advanced into the excluded stomach and duodenum [7]. ERCP is then carried out in standard fashion using standard accessories. EA-ERCP is performed utilizing overtubebased (single balloon, double balloon or spiral) enteroscopy, where the endoscope/overtube combination is passed orally via the Roux limb. Once the enteroenterostomy is reached, the pancreaticobiliary limb is accessed in retrograde fashion in order to reach the papilla [10]. Once the papilla is identified, ERCP is carried out via the forward-viewing, 200-cm-long enteroscope (therapeutic channel $2.8 \mathrm{~mm}$ ) using dedicated "long" accessories. A short version of the double-balloon enteroscope using standard accessories has been investigated but has only recently become available in the United States.

Our meta-analysis suggests that LA-ERCP has significantly higher overall success rates (therapeutic success $97.9 \%$; $95 \%$ Cl: 96.7-98.7\%) than EA-ERCP (therapeutic success $73.2 \%$; $95 \% \mathrm{Cl}: 62.5-82.6 \%)$ at the expense of a higher adverse event rate and longer procedural time. We find that all technical components of ERCP (papilla identification, cannulation and therapeutic success) are more successful with LA-ERCP than EA-ERCP ( $>$ Fig. 1, $>$ Fig. 2, $>$ Fig. 3). The higher papilla identification rate may be explained by the shorter distance the endoscope must traverse to reach the papilla and use of a standard side-viewing duodenoscope in correct orientation with LA-ERCP. Along the same lines, the higher papilla cannulation rates may be ex- 


\section{Study \\ ID}

Percent (95\% Cl)

Emmett, 2007

Itoi, 2011

Schreiner, 2012

Choi, 2013

Shah, 2013

Siddiqui, 2013

Trindade, 2015

De Koning, 2016

Ali, 2018

Kashani, 2018

DL overall $\left(I^{2}=80.2 \%\right)$

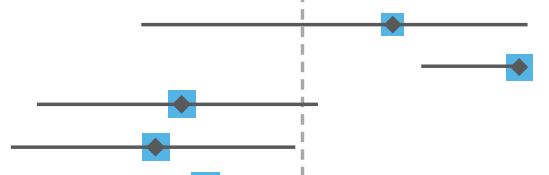

$83.7(54.3,99.2)$

$98.4(86.8,98.6)$

$59.1(42.1,75.0)$

$56.1(39.1,72.3)$

$61.7(49.6,73.2)$

$73.8(59.2,86.0)$

$65.6(51.2,78.6)$

$58.0(38.5,76.3)$

$77.6(61.0,90.6)$

$88.1(82.0,93.1)$

$73.2(62.5,82.6)$

NOTE: Weights are from random effects analysis

$\begin{array}{llllll}0 & 20 & 40 & 60 & 80 & 100\end{array}$

- Fig. 6 Pooled therapeutic success rate of enteroscopy-assisted ERCP arm.

plained by the combination of factors mentioned previously as well as the availability of standard ERCP accessories for use compared to EA-ERCP. Ultimately, the pooled therapeutic success rate with LA-ERCP was remarkably high and is consistent with that of regular ERCP, highlighting that the main limitation of ERCP in RYGB patients is the ability to reach the papilla and then having adequate accessories for use. In contrast, EA-ERCP showed a more modest but heterogenous pooled success rate, with individual studies reporting success rates ranging from $56-98 \%$. Notably, this heterogeneity persisted in subgroup analyses separately assessing different enteroscopy approaches and is unlikely to be attributed solely to the enteroscopy modality utilized. We excluded case series with fewer than five patients to decrease the effect variable operator experience may have on the pooled outcomes, however, residual effects cannot be excluded and may also partially explain the noted heterogeneity.

In line with prior analyses, we found a higher overall AE rate with LA-ERCP. This can mainly be attributed to infectious and bleeding AEs related to the laparoscopic approach of the procedure rather than ERCP. This is supported by our finding that pooled rates of ERCP-related AEs were similar between the two approaches. While many reported laparoscopy-related AEs were self-limited, some were quite serious in nature including bleeding requiring transfusion, intra-abdominal abscess formation and tension pneumothorax. This supports an individualized approach that considers patient comorbidities and characteristics when choosing the most appropriate modality for ERCP.

Expectedly, we also note a shorter mean procedural time with EA-ERCP compared to LA-ERCP. This is readily explained by the additional time required for laparoscopic access to the remnant stomach in LA-ERCP. While the time savings of using EA-ERCP may seem attractive, particularly for busy endoscopy units, this must be weighed against the potential for lower

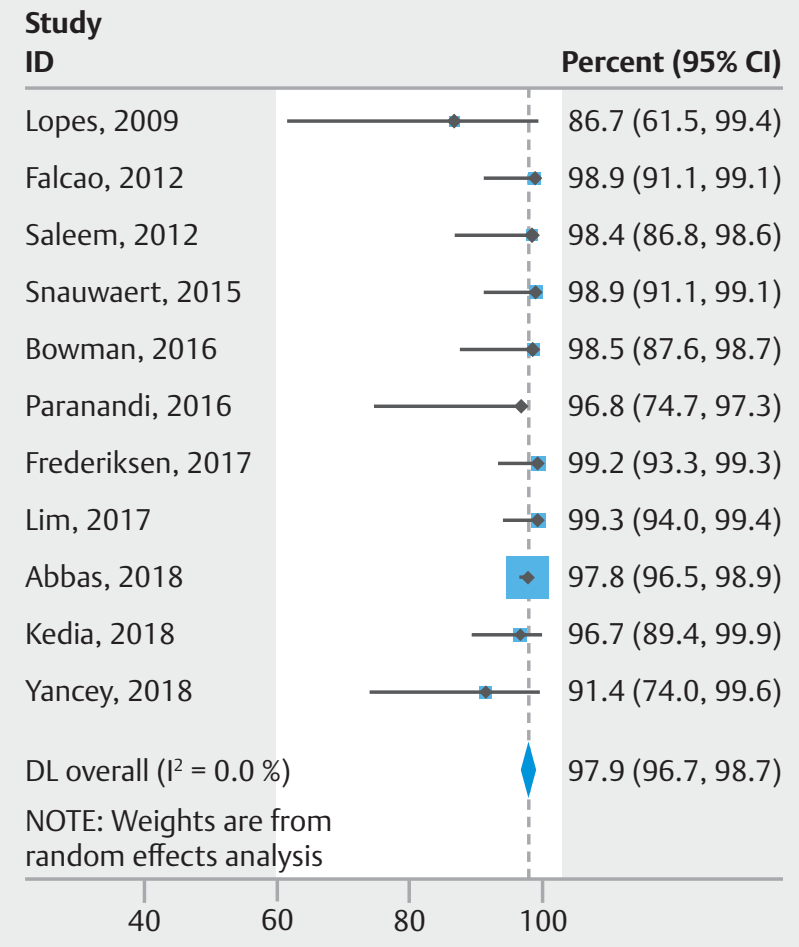

Fig. 7 Pooled therapeutic success rate of laparoscopy-assisted ERCP arm.

overall ERCP success rates compared to LA-ERCP. Notably, a failed attempt at EA-ERCP may inevitably lead to additional interventions such as LA-ERCP or percutaneous transhepatic biliary drainage, each with its associated cost, time, and possible AEs. Interpreting pooled results of procedural time must be 


\section{Study \\ ID \\ Percent (95\% Cl) \\ Emmett, 2007 \\ Saleem, 2010 \\ Itoi, 2011 \\ Schreiner, 2012 \\ Choi, 2013 \\ Siddiqui, 2013 \\ Trindade, 2015 \\ Ali, 2018 \\ Bukhari, 2018 \\ Kashani, 2018 \\ DL overall $\left(I^{2}=16.2 \%\right)$

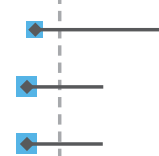 \\ $2.9(2.4,22.7)$ \\ $1.6(1.4,13.2)$ \\ $1.6(1.4,13.2)$ \\ $4.4(0.2,13.9)$ \\ $4.4(0.2,13.9)$ \\ $11.2(3.4,22.7)$ \\ $7.7(1.8,17.2)$ \\ $0.9(0.8,7.4)$ \\ $11.2(2.7,24.5)$ \\ $10.4(5.7,16.2)$ \\ $6.5(3.9,9.6)$ \\ NOTE: Weights are from random effects analysis

$\begin{array}{llll}1 & & 1 & \\ 0 & 20 & 40 & 60\end{array}$

- Fig. 8 Pooled adverse event rate of enteroscopy-assisted ERCP arm. with caution however; only four of 12 EA-ERCP studies reported procedural time with heterogenous underlying enteroscopy modalities and operator experience.

Our study has several strengths. While the higher therapeutic success rate noted with LA-ERCP $(97.9 \%)$ compared to EA$\operatorname{ERCP}(73.2 \%)$ is in line with other systematic reviews on the topic, we attempted to address some of the limitations of other analyses. Recently, Aiolfi et al. reported a pooled LA-ERCP success rate of $99 \%$ in patients with RYGB anatomy [35], however this was limited by the lack of a clear definition for "ERCP success." We utilized strict definitions and we calculated detailed pooled outcomes for papilla identification, cannulation and therapeutic success, respectively. Ponte-Neto et al. recently compared LA-ERCP to balloon-based ERCP, with similar findings to our analysis [36], however, the power of the pooled rate of LA-ERCP success might have been limited by lack of inclusion of the largest multi-center study to date by Abbas et al. which reported outcomes of LA-ERCP in 567 patients from 34 centers [8]. Additionally, The Ponte-Neto analysis limited the enteroscopy arm to balloon-based enteroscopy while we also include studies describing rotational spiral enteroscopy. Finally, by focusing on patients with bariatric RYGB anatomy we aimed to reduce heterogeneity attributed to variable Roux limb length and presence of bilio-enteric anastomoses that may have affected other analyses that include patients with different anatomic variations such as Billroth I, Billroth II, Roux-en-Y hepaticojejunostomy or pancreaticoduodenectomy [37, 38].

Our study has several limitations. The EA-ERCP arm included different enteroscopy modalities including single-balloon, dou-

\section{Study}

ID

Guiterrez, 2009

Lopes, 2009

Bertin, 2011

Falcao, 2012

Saleem, 2012

Grimes, 2015

Snauwaert, 2015

Bowman, 2016

Paranandi, 2016

Frederiksen, 2017

Lim, 2017

Abbas, 2018

Kedia, 2018

Yancey, 2018

DL overall $\left(I^{2}=74.1 \%\right)$

NOTE: Weights are from random effects analysis

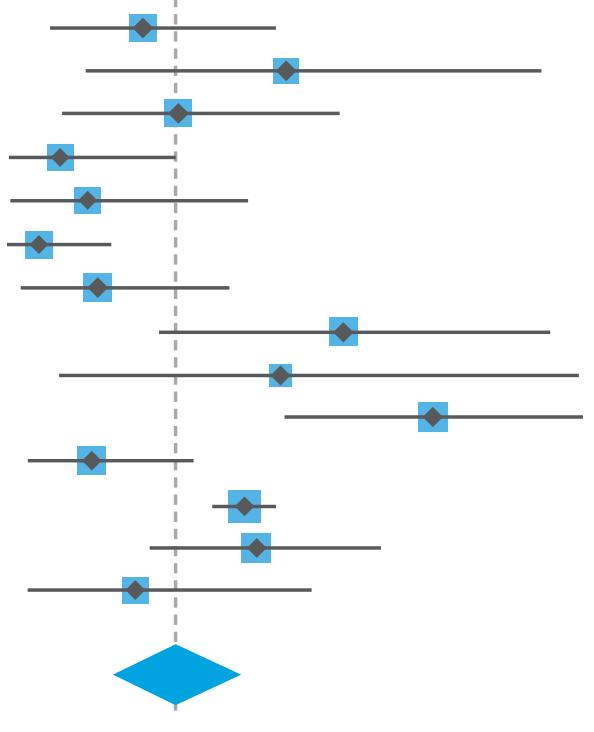

$15.5(4.9,30.6)$

$31.7(8.9,60.8)$

$19.5(6.3,37.8)$

$6.1(0.2,18.9)$

$9.1(0.4,27.5)$

$3.7(0.1,11.9)$

$10.3(1.6,25.3)$

$38.2(17.3,61.8)$

$31.1(5.9,65.0)$

$48.4(31.6,65.5)$

$9.7(2.3,21.3)$

$27.0(23.5,30.7)$

$28.4(16.2,42.5)$

$14.6(2.4,34.7)$

$19.0(12.6,26.4)$

$\begin{array}{lllll}1 & 1 & 1 & 1 & 100\end{array}$

Fig. 9 Pooled adverse event rate of laparoscopy-assisted ERCP arm. 
ble-balloon and spiral enteroscopy, which may contribute to the noted high degree of heterogeneity of our pooled outcomes, however, we attempted to address this by performing subgroup analyses when data were available. Aside from one prospective case series, the remaining included studies had a retrospective design with the inherent limitations of the retrospective approach. This, however, highlights the limitations of available literature rather than the individual analysis. As noted above and inherent to the meta-analytic technique, not every study reported all outcomes of interest and as such, not all studies were included in subgroup analyses when this was the case. Finally, other emerging endoscopic approaches exist which we did not include in our analysis such as endoscopic ultrasound-directed transgastric ERCP (EDGE). Expertise in EDGE remains limited to select centers, but data suggests success rates comparable to LA-ERCP [39].

Ultimately, choice of the optimal ERCP modality in patients with RYGB is dependent on multiple factors including patient preference, indications for ERCP, clinical importance of preserving the integrity of the RYGB, local expertise, and device availability. Based on our current understanding and available data, we suggest the following approach. LA-ERCP can be considered the preferred modality when a single ERCP is likely to address the clinical problem (e.g. choledocholithiasis, papillary stenosis) or when cholecystectomy is indicated thus allowing the ERCP and the cholecystectomy to be carried out in the same session. EDGE may be considered when preserving the integrity of the RYGB is of no clinical significance (e.g. pancreatic head mass likely to be cancer in need of sampling and stenting) or when multiple ERCPs are anticipated (e.g. endoscopic therapy for benign biliary stricture or chronic pancreatitis). Considering the significantly lower success rates, EA-ERCP should be reserved for situations in which it is the only available modality or for patients not willing to undergo LA-ERCP or EDGE.

\section{Conclusion}

In summary, this meta-analysis suggests that LA-ERCP should be considered a first-line approach for ERCP in patients with RYGB due to its higher overall success rate compared to EAERCP. However, LA-ERCP is associated with a higher burden of $A E s$ and longer procedural time. In the absence of high-quality comparative studies, the choice between LA-ERCP and EA-ERCP must be made on a case-by-case basis that takes patient, facility, and endoscopist characteristics into account.

\section{Competing interests}

The authors declare that they have no conflict of interest.

\section{References}

[1] Stevens GA, Singh GM, Lu Y et al. National, regional, and global trends in adult overweight and obesity prevalences. Popul Health Metr 2012; 10: 22
[2] Nudel J, Sanchez VM. Surgical management of obesity. Metabolism 2019; 92: 206-216

[3] Higa KD, Boone KB, Ho T. Complications of the laparoscopic Roux-enY gastric bypass: 1,040 patients - what have we learned? Obes Surg 2000; 10: 509-513

[4] Shuster D, Elmunzer BJ. What is the preferred approach to performing endoscopic retrograde cholangiopancreatography in patients with Roux-en-Y gastric bypass anatomy? Gastroenterology 2014; 146: 1123-1125

[5] Frederiksen NA, Tveskov L, Helgstrand F et al. Treatment of Common bile duct stones in gastric bypass patients with laparoscopic transgastric endoscopic retrograde cholangiopancreatography. Obes Surg 2017; 27: 1409-1413

[6] Schreiner MA, Chang L, Gluck M et al. Laparoscopy-assisted versus balloon enteroscopy-assisted ERCP in bariatric post-Roux-en-Y gastric bypass patients. Gastrointest Endosc 2012; 75: 748-756

[7] Doshi B, Yasuda I, Ryozawa S et al. Current endoscopic strategies for managing large bile duct stones. Dig Endosc 2018; 30: 59-66

[8] Abbas AM, Strong AT, Diehl DL et al. Multicenter evaluation of the clinical utility of laparoscopy-assisted ERCP in patients with Roux-en-Y gastric bypass. Gastrointest Endosc 2018; 87: 1031-1039

[9] Bertin PM, Singh K, Arregui ME. Laparoscopic transgastric endoscopic retrograde cholangiopancreatography (ERCP) after gastric bypass: Case series and a description of technique. Surg Endosc 2011; 25: 2592-2596

[10] Ali MF, Modayil R, Gurram KC et al. Spiral enteroscopy-assisted ERCP in bariatric-length Roux-en-Y anatomy: a large single-center series and review of the literature (with video). Gastrointest Endosc 2018; 87: $1241-1247$

[11] Abu Dayyeh B. Single-balloon enteroscopy-assisted ERCP in patients with surgically altered $\mathrm{Gl}$ anatomy: getting there. Gastrointest Endosc 2015; 82: 20-23

[12] Wells GA, Shea B, Connell D et al. The Newcastle-Ottawa Scale (NOS) for assessing the quality of nonrandomised studies in meta-analyses. Ottawa Hosp Res Inst 2000: 2-4

[13] Nyaga VN, Arbyn M, Aerts M. Metaprop: A Stata command to perform meta-analysis of binomial data. Arch Public Heal 2014; 72: 1-10

[14] Bowman E, Greenberg J, Garren M et al. Laparoscopic-assisted ERCP and EUS in patients with prior Roux-en-Y gastric bypass surgery: a dual-center case series experience. Surg Endosc 2016; 30: 46474652

[15] De Koning M, Moreels TG. Comparison of double-balloon and singleballoon enteroscope for therapeutic endoscopic retrograde cholangiography after Roux-en-Y small bowel surgery. BMC Gastroenterol 2016; 16: 98

[16] Emmett DS, Mallat DB. Double-balloon ERCP in patients who have undergone Roux-en-Y surgery: a case series. Gastrointest Endosc 2007; 66: 1038-1041

[17] Falcão M, Campos JM, Neto MG et al. Transgastric endoscopic retrograde cholangiopancreatography for the management of biliary tract disease after Roux-en-Y gastric bypass treatment for obesity. Obes Surg 2012; 22: 872-876

[18] Grimes KL, Maciel VH, Mata W et al. Complications of laparoscopic transgastric ERCP in patients with Roux-en-Y gastric bypass. Surg Endosc 2015; 29: 1753-1759

[19] Gutierrez JM, Lederer H, Krook JC et al. Surgical gastrostomy for pancreatobiliary and duodenal access following Roux en $\mathrm{Y}$ gastric bypass. J Gastrointest Surg 2009; 13: 2170-2175

[20] Itokawa F, Ishii K, Tanaka R et al. Large balloon dilatation following endoscopic sphincterotomy using a balloon enteroscope for the bile duct stone extractions in patients with Roux-en-Y anastomosis. Dig Liver Dis 2011; 43: 237-241 
[21] Kashani A, Abboud G, Lo SK et al. Double balloon enteroscopy-assisted endoscopic retrograde cholangiopancreatography in Roux-en-Y gastric bypass anatomy: expert vs. novice experience. Endosc Int open 2018; 6: E885-E891

[22] Lim CH, Jahansouz C, Freeman ML et al. Outcomes of endoscopic retrograde cholangiopancreatography (ercp) and sphincterotomy for suspected sphincter of Oddi dysfunction (SOD) post Roux-en-Y gastric bypass. Obes Surg 2017; 27: 2656-2662

[23] Lopes TL, Clements RH, Wilcox CM. Laparoscopy-assisted ERCP: experience of a high-volume bariatric surgery center (with video). Gastrointest Endosc 2009; 70: 1254-1259

[24] Paranandi B, Joshi D, Mohammadi B et al. Laparoscopy-assisted ERCP (LA-ERCP) following bariatric gastric bypass surgery: Initial experience of a single UK centre. Frontline Gastroenterol 2016; 7: 54-59

[25] Petersen B, Saleem A, Levy M et al. Endoscopic retrograde cholangiopancreatography using a single-balloon enteroscope in patients with altered Roux-en-Y anatomy. Endoscopy 2010; 42: 656-660

[26] Saleem A, Levy MJ, Petersen BT et al. Laparoscopic assisted ERCP in Roux-en-Y gastric bypass (RYGB) surgery patients. J Gastrointest Surg 2012; 16: 203-208

[27] Shah RJ, Smolkin M, Yen R et al. A multicenter, U.S. experience of single-balloon, double-balloon, and rotational overtube-assisted enteroscopy ERCP in patients with surgically altered pancreaticobiliary anatomy (with video). Gastrointest Endosc 2013; 77: 593-600

[28] Siddiqui AA, Chaaya A, Shelton C et al. Utility of the short doubleballoon enteroscope to perform pancreaticobiliary interventions in patients with surgically altered anatomy in a US multicenter study. Dig Dis Sci 2013; 58: 858-864

[29] Snauwaert C, Laukens P, Dillemans B et al. Laparoscopy-assisted transgastric endoscopic retrograde cholangiopancreatography in bariatric Roux-en-Y gastric bypass patients. Endosc Int Open 2015; 3: E458-E463
[30] Trindade A, Mella J, Slattery E et al. Use of a cap in single-balloon enteroscopy-assisted endoscopic retrograde cholangiography. Endoscopy 2014; 47: 453-456

[31] Habenicht YanceyK, McCormack LK, McNatt SS et al. Laparoscopicassisted transgastric ERCP: a single-institution experience. J Obes 2018; 2018: 8275965

[32] Choi EK, Chiorean MV, Coté GA et al. ERCP via gastrostomy vs. double balloon enteroscopy in patients with prior bariatric Roux-en-Y gastric bypass surgery. Surg Endosc 2013; 27: 2894-2899

[33] Bukhari M, Kowalski T, Nieto J et al. An international, multicenter, comparative trial of EUS-guided gastrogastrostomy-assisted ERCP versus enteroscopy-assisted ERCP in patients with Roux-en-Y gastric bypass anatomy. Gastrointest Endosc 2018; 88: 486-494

[34] Kedia P, Tarnasky PR, Nieto J et al. EUS-directed transgastric ERCP (EDGE) versus laparoscopy-assisted ERCP (LA-ERCP) for Roux-en-Y gastric bypass (RYGB) anatomy. J Clin Gastroenterol 2019; 53: 304308

[35] Aiolfi A, Asti E, Rausa E et al. Trans-gastric ERCP after Roux-en-Y gastric bypass: systematic review and meta-analysis. Obes Surg 2018; 28: 2836-2843

[36] da Ponte-Neto AM, Bernardo WM, de A Coutinho LM et al. Comparison between enteroscopy-based and laparoscopy-assisted ERCP for accessing the biliary tree in patients with Roux-en-Y gastric bypass: systematic review and meta-analysis. Obes Surg 2018; 28: 40644076

[37] Skinner M, Popa D, Neumann H et al. ERCP with the overtube-assisted enteroscopy technique: A systematic review. Endoscopy 2014; 46: 560-572

[38] Inamdar S, Slattery E, Sejpal DV et al. Systematic review and meta-analysis of single-balloon enteroscopy-assisted ERCP in patients with surgically altered GI anatomy. Gastrointest Endosc 2015; 82: 9-19

[39] Wang T], Ryou M. Evolving techniques for endoscopic retrograde cholangiopancreatography in gastric bypass patients. Curr Opin Gastroenterol 2018; 34: 444-450 